\title{
A New Parameter to Describe Light Scattering by an Arbitrary Sphere
}

\author{
William R. Heinson, Amitabha Chakrabarti and Christopher M. Sorensen* \\ Department of Physics, Kansas State University, 116 Cardwell Hall, Manhattan, KS 66506-2601 \\ *Corresponding author: sor@phys.ksu.edu
}

Received Month X, XXXX; revised Month X, XXXX; accepted Month X, XXXX; posted Month X, XXXX (Doc. ID XXXXX); published Month X, XXXX

\begin{abstract}
We consider light scattering by uniform spheres of arbitrary size $\mathrm{R}$ and refractive index $\mathrm{m}$. A new internal coupling parameter $\rho^{\prime}=2 k R\left|\left(m^{2}-1\right) /\left(m^{2}+2\right)\right|$ where $k=2 \pi / \lambda$ and $\lambda$ is the wavelength is proposed that has a superior ability to determine the asymptotic regimes of small size, weakly refractive and large size, refractive than the parameter $\rho=2 k R(m-1)$. It also has superior ability as a parameter to organize patterns in light scattering from spheres.

Key words: Scattering; Mie scattering; diffraction limits.
\end{abstract}

\section{INTRODUCTION}

The Mie solution for how electromagnetic radiation such as light scatters from spheres of arbitrary size and refractive index was presented more than 100 years ago[1]. The physical problem of light scattering has two limits: the small size or weakly refractive limit in which the scattering is described by three-dimensional Fraunhofer diffraction; and the large size, refractive limit in which scattering is described by two-dimensional Fraunhofer diffraction from the projected geometric cross section of the object. Given this physical perspective, we expect that the Mie solution will yield these two diffraction patterns in their corresponding limits. The purpose of this paper is to establish the correct parameter to quantitatively determine the limits of either small size or weakly refractive or large size and refractive. We will refer to these as the Rayleigh-Debye-Gans (RDG) and geometric limits, respectively.

\section{ANALYSIS}

To study the scattering we will examine the angular dependence of the scattered intensity, I. From this perspective, it is very useful to forgo the usual scattering angle $\theta$ functionality and use instead the magnitude of the scattering wave vector [2-4]

$$
q=2 k \sin (\theta / 2)
$$

where $k=2 \Pi / \lambda$ and $\lambda$ is the wavelength of light. We have come to call the method of plotting the scattered intensity versus $q$ on a double logarithmic plot "Q-space analysis" [4].

The RDG (small size or weakly refractive) limit for Mie scattering can be quantitatively specified by the so-called phase shift parameter $\rho=2 k R(m-1)$

For a sphere of any radius $R$ and refractive index $m$, experiencing an incident intensity of unity, under the condition $\rho \rightarrow 0$, the RDG scattered intensity is [5-7]

$I(q)=I_{R}\left[\left(3 / u^{3}\right)(\sin u-u \cos u)\right]^{2}$

where

$I_{R}=k^{4} R^{6}\left|\frac{\left(m^{2}-1\right)}{\left(m^{2}+2\right)}\right|^{2}$

and

$u=q R$

We use the term " $I_{R}$ " for the scattering that would come from a particle in the Rayleigh limit, $k R \ll 1$ and $|m| k R \ll 1$. It is identical to the forward scattering intensity $I(0)=I_{R}$ whenever $\rho \rightarrow 0$. When $\rho>1$, the full Mie result becomes necessary.

Note that the refractive index functionality is the square of the Lorentz-Lorenz term which is the dynamic embodiment of the Clausius-Mossotti relation [8]. Equations (3) through (5) describe the Fraunhofer diffraction from a three-dimensional sphere.

The physical basis for the RDG limit is that the scattering particle's interior field becomes the incident field. Then in this limit each sub-volume $\mathrm{dV}$ of the particle acts like a Rayleigh scatterer scattering with a scattered field proportional to the incident field and $\left[\left(m^{2}-1\right) /\left(m^{2}+2\right)\right] d V$. In the forward direction all these fields add in phase to yield $\mathrm{I}_{\mathrm{R}}$, the first term in Eq. (3). At other angles, phase differences lead to the second term in Eq. (3). Important for this limit, however, is that these sub- 
volumes are affected by only the incident field and not the scattered fields from the rest of the particle. Said differently, the internal coupling between different parts of the particle is weak.

This physical basis lies at the foundation of the argument presented by van de Hulst who, in section 6.22 of his book [5], assumed the polarization of a solid particle induced by an applied electric field does not affect the interior field of the particle in the $m \rightarrow 1$ limit. Had he allowed the induced polarization to feed back to the particle the well-known ClausiusMossotti relation would have been obtained. Subsequently, at the beginning of chapter 7 , he introduces the expression for $\rho$ as the governing parameter to determine when the RDG limit applies. Note that the Lorentz-Lorenz term reduces to the $\mathrm{m}-1$ functionality of $\rho$ in the $m \rightarrow 1$ limit. It is also here that he uses the term "phase shift", but it is not until section 10.1 that he states that $\rho$ is equal to the phase difference between a wave passing through a diameter of the particle and one traveling the same distance outside the particle. Kerker, in chapter 8 of his book [6], explicitly writes the coupling fields from particle subvolumes in terms of the Lorentz-Lorenz relation, but before he applies that, he makes the approximation $m \rightarrow 1$ and, as for van de Hulst, claims that $\rho$ is the governing parameter to determine the small size, weakly refractive limit.

In light of this history and given the physical basis for the RDG limit, it is worthwhile to ask if a modified parameter based on the Clausius-Mossotti/Lorentz-Lorenz relations might be a more appropriate parameter to describe the limits of Mie scattering. This question is answered by considering the other limit of a large, refractive particle, the geometric limit.

For a circular obstacle of radius $R$ and "sufficiently large" (a term to be described below) refractive index, experiencing an incident intensity of unity, the Fraunhofer diffraction pattern is [9]

$I(q)=\left(k^{2} R^{4} / 4\right)\left[\frac{2 J_{1}(u)}{u}\right]^{2}$

In (6) $J_{1}(u)$ is the first Bessel function of the first kind. We expect that this is also the scattering from a sphere in the geometric limit. This has been recently demonstrated for the Mie formulation [10]. Then the forward scattering is $I(0)=k^{2} R^{4} / 4$.

In previous work [3] we have shown that for spheres with $\rho \geq 10$ the forward scattering goes as

$I(0) \simeq 3 I_{R} / \rho^{2}$

We have also studied the approach of Mie scattering to the geometric, two-dimensional Fraunhofer limit at large $\rho$ [10]. In principle, the forward scattering at extremely large $\rho$ should be identical to the two-dimensional result, the first term of Eq. (6), $I(0)=k^{2} R^{4} / 4$. However, Eq. (7) is incapable of achieving this limit because the refractive index functionalities do not cancel. If, however, one defines a modified phase shift parameter, $\rho^{\prime}$, such that

$\rho^{\prime}=2 k R\left|\frac{m^{2}-1}{m^{2}+2}\right|$ then no refractive index dependency remains and Eq. (7) can be modified to

$I(0)=I_{R} / \rho^{\prime 2}$

for $\rho^{\prime} \geq 10$. This yields the desired result $I(0)=k^{2} R^{4} / 4$.

Since $\rho$ ' is derived using the Lorentz-Lorenz term which is a measure of how strong the internal coupling between different sub-volumes of the particle is, we henceforth call $\rho$ ' the "internal coupling parameter".

\section{TESTS OF THE ANALYSIS}

Scattering data were calculated using Philip Laven's freeware program MiePlot [11]. MiePlot is a simple yet robust interface built to use the Bohren and Huffman algorithm for Mie scattering.

Figure 1a shows plots of the forward scattered intensity, I(0), normalized by the Rayleigh scattered intensity, Eq. (4), (which we will refer to as the "Rayleigh normalized forward scattering intensity") versus the phase shift parameter $\rho$. A quasi-universal dependence on $\rho$ is seen despite the wide variation on the refractive index [3]. This supports the contention of Eq. (7). In contrast Fig. 1b plots the Rayleigh normalized forward scattering intensity versus the internal coupling parameter $\rho$ '. Now, at large $\rho$ ', all the plots fall together on the same line. More resolution for this comparison can be gained by multiplying the Rayleigh normalized scattering intensity by either $\rho^{2}$ or $\rho^{2}$ as done in Figs. 1c and 1d, respectively. While Fig. $1 c$ shows differences on the order of a factor of 2 at large $\rho$, the plots using $\rho$ ' in Fig. 1d fall accurately together at large $\rho$ '. These results support the contention that $\rho$ ' is an accurate parameter to determine when Mie scattering is entering the large size, refractive regime. They also indicate that $\rho$ ' can be used to unify quantitatively the forward scattering in that regime to a single description.

In previous work $\rho$ was shown to provide a quasiuniversal description of the entire Mie scattering angular dependence of the scattered intensity. We now test the new parameter $\rho$ ' for a similar description and compare it to $\rho$ in Fig. 2 . There we find the Rayleigh normalized scattering intensity plotted versus qR on a log-log plot; a method we call Q-space analysis [4]. The intensities have been averaged over a lognormal size distribution with geometric standard deviation of 1.2 to remove the ripple structure. This leaves smooth curves that represent the general functionalities of the scattering. Figure $2 \mathrm{a}$ shows that for a given $\rho$ value, the curves show approximately the same functional dependence despite the wide range of sizes and refractive indices. On the other hand, the plots for the two different $\rho$ values are distinctively different. This is the quasiuniversality with $\rho$ as the organizing parameter alluded to above. In contrast Fig. $2 b$ shows that for a given $\rho$ ' value the curves overlap much more closely than for $\rho$. This implies that the patterns unveiled by $Q$-space analysis for Mie scattering are well parameterized by the internal coupling parameter $\rho$ ' and this provides a better organization than the phase shift parameter $\rho$. We conclude that spheres with the same value of $\rho$ ' exhibit very similar scattering regardless of size $\mathrm{R}$ and refractive index $\mathrm{m}$. 
However, return to Fig. 1 for the $1<\rho<10$ range where crossover ripples dominate. In this range $\rho$ appears to coordinate

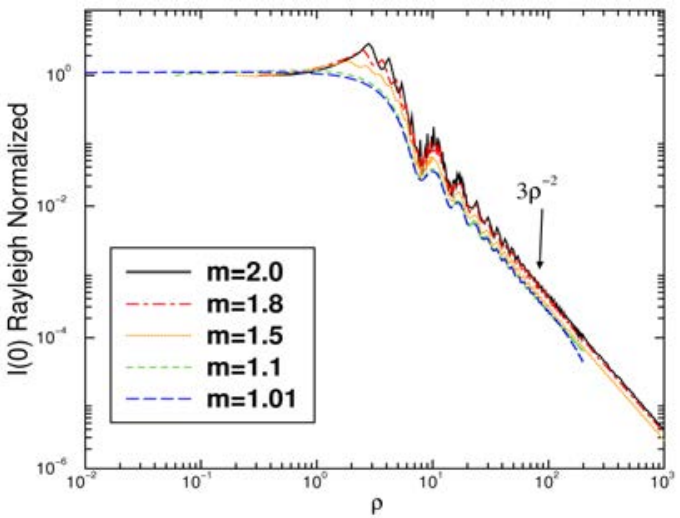

a.

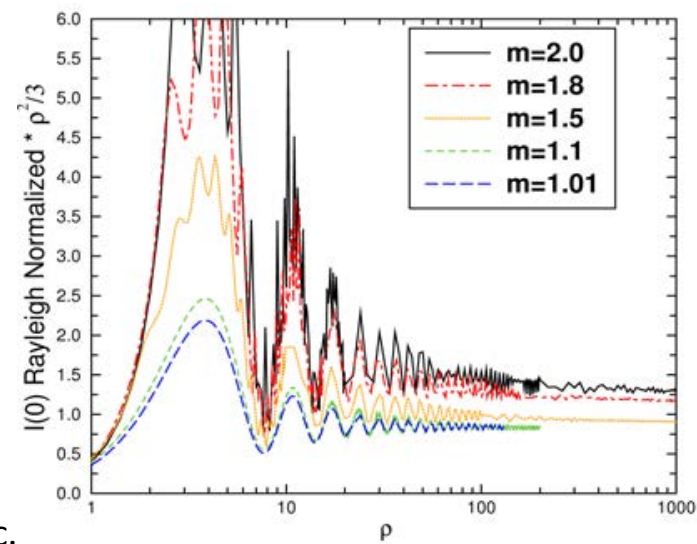

the phase of the ripples better than $\rho$ '.
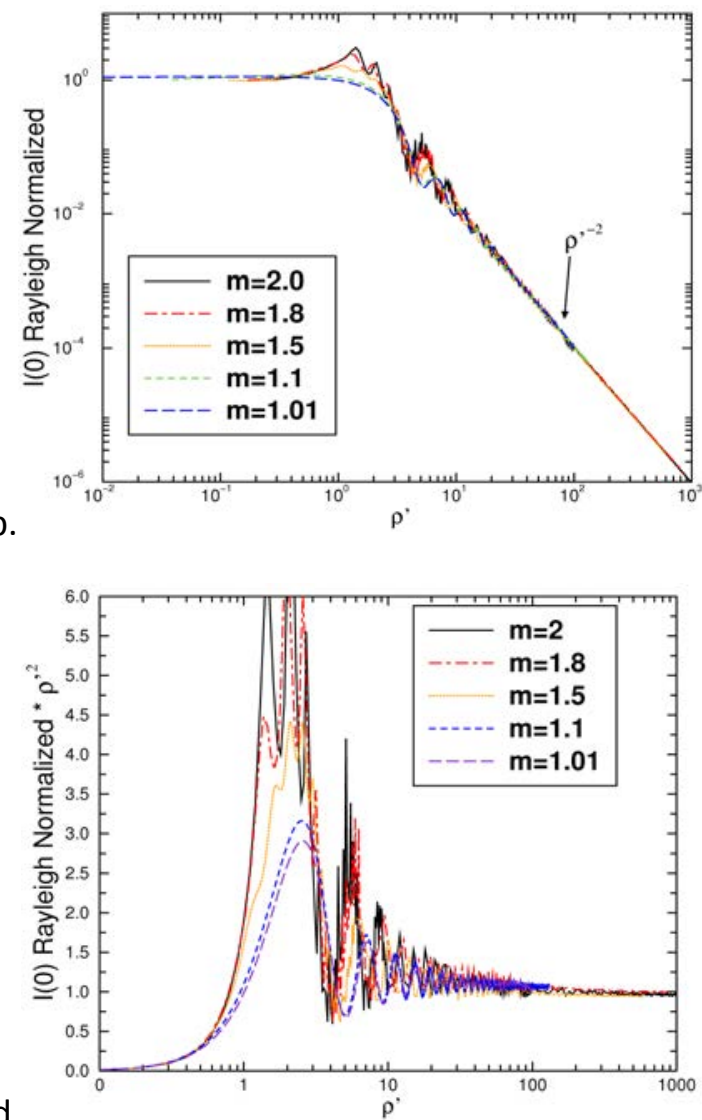

Figure 1 (a) Rayleigh normalized forward scattering versus the phase shift parameter $\rho$ with various refractive indexes m. (b) Rayleigh normalized forward scattering versus the internal coupling parameter $\rho$ ' with various refractive indexes. (c) Data from (a) multiplied by $\rho^{2} / 3$. (d) Data from (b) multiplied by $\rho^{\prime 2}$. As spheres reach the geometric limit, plotting with $\rho$ still shows refractive index dependency while plotting versus $\rho$ ' does not.

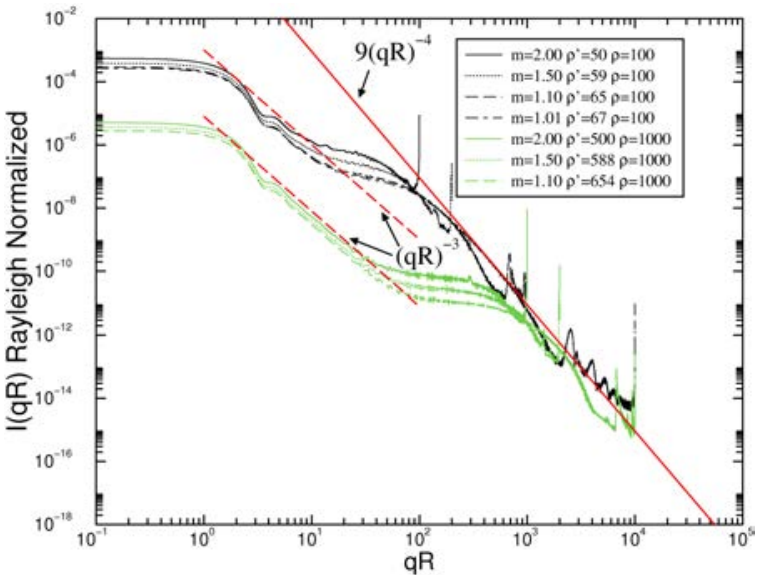

a.

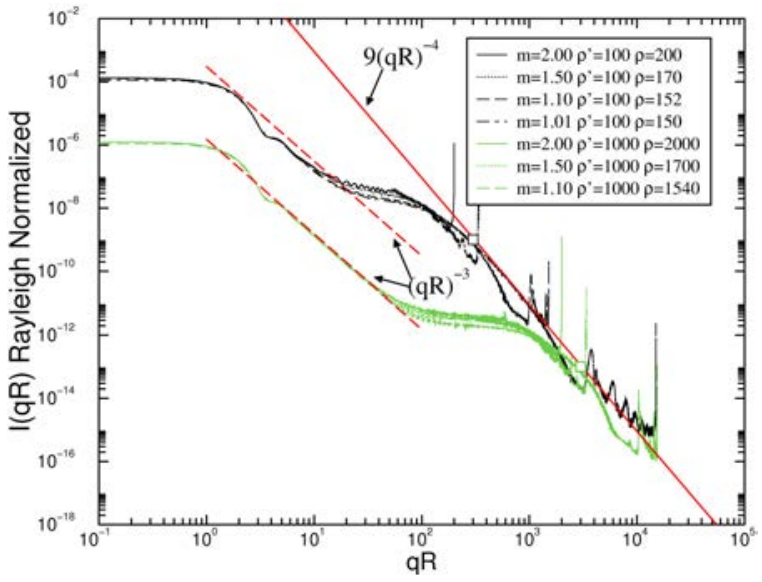

b.

Figure 2 (a) Rayleigh normalized scattering from polydisperse spheres with various mean sizes and refractive indices combined to yield phase shift parameters of either $\rho=100$ or 1000 mean values. (b) Rayleigh normalized scattering from polydisperse spheres with various mean sizes and refractive indices combined to yield internal coupling parameters of either $\rho^{\prime}=100$ or 1000 mean values. The line of $9(q R)^{-4}$ is the envelope of scattering from a Rayleigh-Debye-Gans (RDG) spherical particle. Lines of $(\boldsymbol{q} \boldsymbol{R})^{-3}$ indicate the Porod regimes of $2 \mathrm{~d}$ circular aperture diffraction. 
Figure 3 shows a comparison of the two parameters. Approximately $\rho^{\prime}=\rho / \sqrt{3}$.
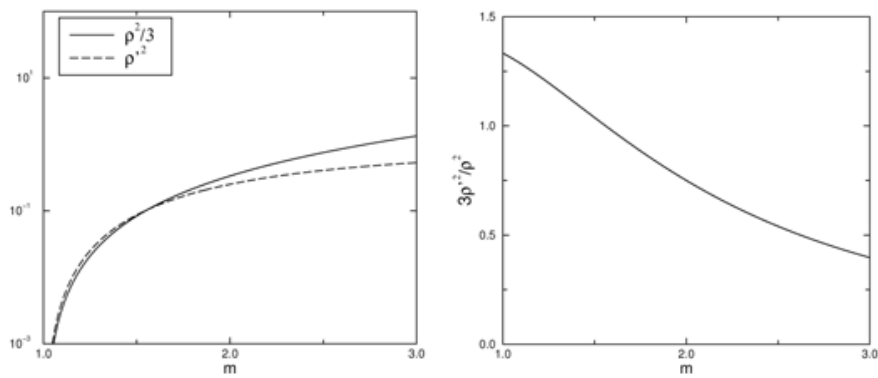

b.

Figure 1 (a) $\rho^{2} / 3$ and $\rho^{\prime 2}$ versus $m$ with $2 k R=1$. (b) The ratio between $\rho^{2} / 3$ and $\rho^{\prime 2}$ versus $m$.

\section{CONCLUSION}

Because the Mie equations for scattering by an arbitrary sphere are based on the fundamental Maxwell equations, it should contain diffraction by both a threedimensional sphere and by a two-dimensional circular object in the RDG and geometric limits, respectively, and indeed the equations do. The physical basis for the RDG limit is that the particle's internal field is not affected by internal coupling between different parts of the particle; hence the internal field is the incident field. Because the scattering from a sub-volume of the particle is proportional to $\left|\left(m^{2}-1\right) /\left(m^{2}+2\right)\right| d V$, it is expected that the most accurate description of the refractive index functionality for the internal coupling is $\mid\left(m^{2}-1\right) /\left(m^{2}+\right.$ $2) \mid$, the Lorentz-Lorenz term. This conclusion is supported by the empirical results reported here. These results allow us to defined a new parameter $\rho$ ' that we call the internal coupling parameter. Moreover, we find that the internal coupling parameter accurately describes the other limit in the large size, physical optics regime and the general behavior of Mie scattering for any sphere between these limits.

Funding Information

National Science Foundation (NSF) AGM 1261651; Army

Research laboratory (AR L) W911NF-14-1-0352.

\section{REFERENCES}

1. G. Mie, "Beitrage zur Optik truber Medien speziel kolloidaler Metallosungen," Ann. Phys. 25, 377-445 (1908).

2. C. M. Sorensen, D. F. Fischbach, "Patterns in Mie Scattering", Opt. Commun. 173, 145-153 (2000).

3. M.J. Berg, C.M. Sorensen, and A. Chakrabarti, "Patterns in Mie Scattering: Evolution when Normalized by the Rayleigh Cross Section," Applied Optics 44, 7487-7493 (2005).

4. C. M. Sorensen, "Q-Space Analysis of Scattering by Particles: A Review”, J. Quant. Spect. Rad. Trans., 131, 312 (2013).

5. H. C. van de Hulst, Light Scattering by Small Particles (Dover, 1981)
6. M. Kerker, The Scattering of Light and Other Electromagnetic Radiation (Academic Press, New York, 1969).

7. C. F. Bohren and D. R. Huffman, Absorption and Scattering of Light by Small Particles (Wiley, New York, 1983).

8. J. D. Jackson, Classical Electrodynamics (Wiley, New York, 1962) pp. 117 to 119.

9. E. Hecht, Optics, 2nd Ed., (Addison-Wesley, Reading MA, 1987).

10. W. R. Heinson, A. Chakrabarti and C. M. Sorensen, "Crossover from spherical particle Mie scattering to circular aperture diffraction”, J. Opt. Soc. Am. A31, 236264 (2014).

11. P. Laven, "Simulation of rainbows, coronas, and glories by use of Mie theory," Appl. Opt. 42, 436-444 (2003). 